\title{
MIXED LAYER DEPTHS VIA DOPPLER LIDAR DURING LOW-LEVEL JET EVENTS
}

\author{
Brian Carroll $^{1 *}$, Belay Demoz ${ }^{1}$, Timothy Bonin ${ }^{2}$, and Ruben Delgado ${ }^{1}$ \\ ${ }^{1}$ Joint Center for Earth Systems Technology/Univ. of Maryland, Baltimore County, MD, USA \\ *brian.carroll@umbc.edu \\ ${ }^{2}$ Cooperative Institute for Research in Environmental Sciences, Boulder, CO, USA
}

\begin{abstract}
A low-level jet (LLJ) is a prominent wind speed peak in the lower troposphere. Nocturnal LLJs have been shown to transport and mix atmospheric constituents from the residual layer down to the surface, breaching quiescent nocturnal conditions due to high wind shear. A new fuzzy logic algorithm combining turbulence and aerosol information from Doppler lidar scans can resolve the strength and depth of this mixing below the jet. Conclusions will be drawn about LLJ relations to turbulence and mixing.
\end{abstract}

\section{INTRODUCTION}

A low-level jet (LLJ) is a mesoscale phenomenon defined simply as a prominent wind speed peak in the lower troposphere with a significant decrease in wind speed above the peak. LLJs over land are almost exclusively nocturnal events that can persist from sunset to sunrise, reaching peak speeds anywhere from about $12 \mathrm{~m} / \mathrm{s}$ to $30 \mathrm{~m} / \mathrm{s}$ depending on geographic location and environmental conditions. The wind speed peak, referred to as the jet "nose", is typically below 1 $\mathrm{km}$ AGL due to linkages between jet formation mechanisms and the diurnal boundary layer cycle.

These jets transport atmospheric constituents such as water vapor and pollution hundreds of kilometers, and the high wind shear below the jet nose is known to generate substantial mixing during otherwise quiescent nocturnal conditions. This mixing of the residual and surface layers can lead to exceedances of air quality thresholds [1] and impact surface moisture flux. The depth of the mixed layer is also a key parameter for numerical weather prediction.

Continuous and accurate measurement of the mixed layer height, especially at night, is a challenging task. Radiosondes provide only an instantaneous snapshot of atmospheric structure.
Radar wind profilers perform well during daytime convective conditions, but have trouble at night with minimum range potentially higher than a shallow boundary layer. Backscatter lidars perform well during the day, but have trouble discerning the MLH from residual layer depth outside of daytime conditions.

Scanning Doppler lidar has many strengths in approaching the problem of MLH retrieval. It can observe aerosol backscatter profiles and provide profiles of turbulence information such as vertical velocity variance. Multiple methods have been utilized in the literature. Tucker et al. [2] found vertical velocity variance to be the most accurate single measure of MLH from Doppler lidar, but all have their strengths and weaknesses (e.g. minimum range for vertical stares possibly overshooting the MLH at night). A new fuzzy logic algorithm uses all information relating to the mixed layer from a Doppler lidar scan routine to produce one best estimate for the MLH. This algorithm will be used here to study mixing and turbulence linkages to LLJs.

\section{METHODOLOGY}

\subsection{Mixed layer height retrieval algorithm}

Several independent techniques exist for estimating MLH from Doppler lidar, most of which rely on retrieving either proxies for turbulence (i.e. velocity variance) or aerosol presence. Velocity variance methods include vertical velocity variance from vertical stares, variance of velocity-azimuth-display (VAD) fit residuals, and vertical slice scan velocity variance which encompasses variations in horizontal wind. Methods that rely on aerosol presence and gradients in the aerosol profile include the Haar wavelet transform and signal-to-noise ratio variance. Each of these methods has strengths and weaknesses in terms of retrieval accuracy, and any given scan is confined to minimum and maximum 
measurement altitudes. For example, VAD fit residuals from low elevation conical scans can yield high-resolution information at low altitudes but the elevation angle results in rapid signal attenuation in the vertical. This can fill in the gap below a vertical stare's minimum range. In this way, combining all methods of MLH retrieval optimizes vertical range and resolution.

The new MLH algorithm presented here utilizes fuzzy logic to combine the independent methods in two phases. Gravity waves are removed before the bulk of this process by removing lowfrequency variance, as they are indicative of larger-scale motions and not the mixing height. The first phase then fuzzifies each of the velocity variance method profiles, converting each variance measurement to a fuzzy membership value between zero and one via a membership function. This is demonstrated in Fig. 1. The membership value represents the variance in a format that is easily combined with the other independent methods. All membership profiles are interpolated to a $5 \mathrm{~m}$ vertical resolution grid and averaged together to produce an aggregate membership profile. A first guess for the MLH is obtained by finding the lowest altitude bin that has an aggregate membership value $<0.5$, effectively expressing $<50 \%$ likelihood of being in the surface-connected turbulent ML.

The first guess derived from the velocity variance methods is then supplemented by the methods related to aerosol presence and gradients. The aerosol methods use peaks in profiles of Haar wavelet transforms or signal-to-noise ratio variance, that can represent the boundary between mixed layer aerosol and cleaner air above. Aerosol is used as the second method because it is less of a direct measure of mixing and hence less reliable; false boundaries are possible due to residual layers and other effects. To avoid these false peaks, the aerosol method peaks are only allowed to contribute to a final guess of the MLH if they are close to the first guess. Peaks in the wind shear profile are also included in this second phase.

A final best estimate for the MLH is obtained by applying a threshold to the updated aggregate membership profile in an identical manner to the first guess. Upper and lower confidence bounds are then determined by 0.8 and 0.2 thresholds, respectively. This is shown in Fig. 2.
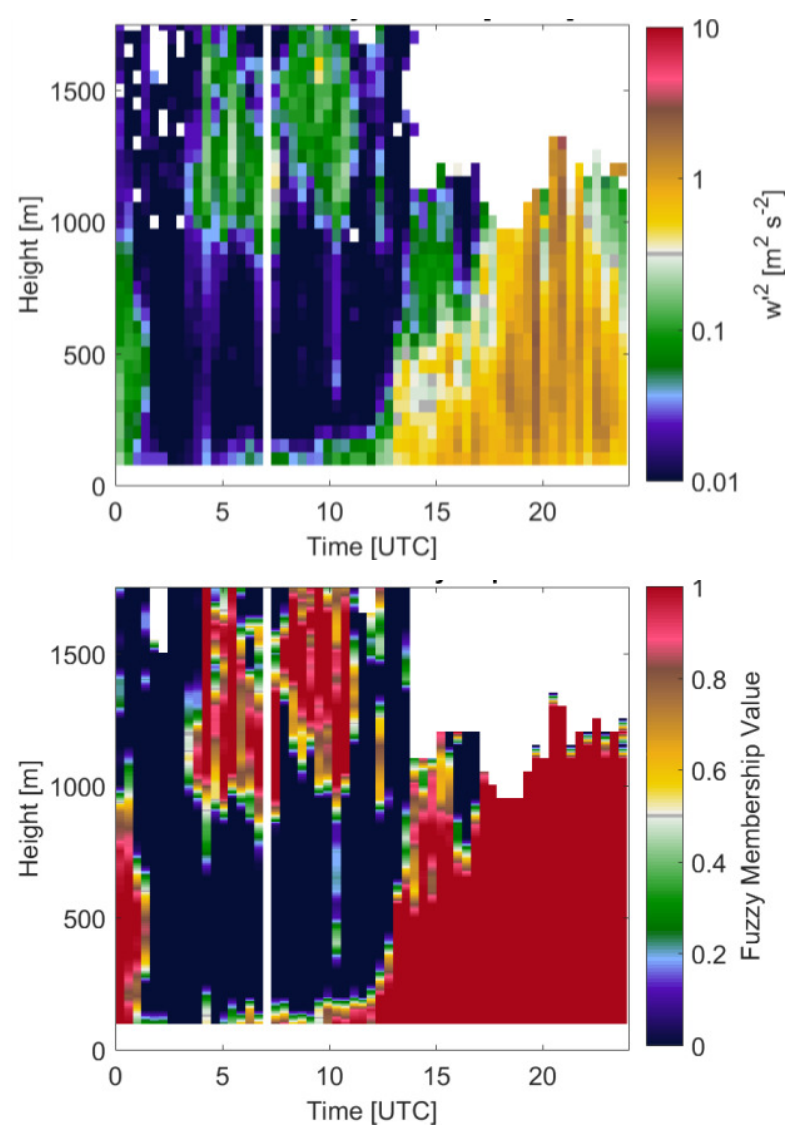

Figure 1: Variance of $45^{\circ}$ elevation angle VAD fit residuals over a 24-hour period (top) and the corresponding fuzzy values (bottom).

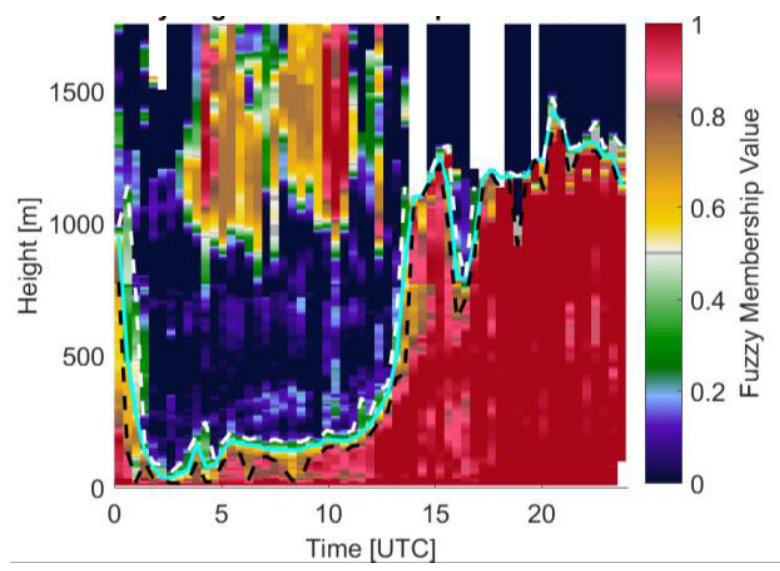

Figure 2: Final aggregate membership profiles for the same period as Fig. 1. Retrieved MLH is the cyan line, with upper and lower confidence bounds as white and black dashed lines, respectively. 


\subsection{Measurements}

The data for this study is from the Plains Elevated Convection at Night (PECAN) campaign, which was a large multi-agency project targeting warmseason nocturnal precipitation and related phenomena over the Great Plains region of the Unites States. The Great Plains LLJ can generate or amplify mesoscale convective systems and has a large impact on the region's moisture budget [3]. It also modulates air quality via mixing as discussed previously [4]. PECAN measurements were taken from June $1^{\text {st }}$ to July $15^{\text {th }}, 2015$.

The scanning Doppler lidar used for MLH retrieval during PECAN was a Leosphere WINDCUBE ${ }^{\text {TM }} 200$ S owned by the University of Maryland, Baltimore County. It ran almost continuously for the six weeks of PECAN. The scan cycle period was approximately 25 minutes long and had a vertical stare, 5 conical scans between $5^{\circ}$ and $45^{\circ}$ elevation, and two orthogonal vertical slice scans. The lidar was co-located with several other instruments, including an atmospheric emitted radiance interferometer (AERI), radiosondes, and surface instruments. The MLH algorithm shows good agreement with the height of temperature inversions retrieved from PECAN radiosondes.

\section{PRELIMINARY RESULTS}

A VAD wind retrieval and the MLH algorithm have been applied to the Doppler lidar PECAN dataset to examine LLJs and their interaction with the boundary layer and turbulence. 33 LLJs were observed with a wide range of wind speed and nose height.

Current preliminary results qualitatively show some major differences in mixing below different LLJs. Two jets of very similar peak wind speed and nose height are shown in Fig. 3, plotted along with the retrieved MLH. Despite similar shear profiles below the jet nose, the June $20^{\text {th }}$ case has little nocturnal mixing but the June $21^{\text {st }}$ case shows substantial surface-connected mixing up to about $160 \mathrm{~m} \mathrm{AGL}$. The larger shear above the nose on June $21^{\text {st }}$ may contribute to this, affecting the stability of the whole layer.

Static stability will be investigated as a key parameter to further this study. Static stability and turbulence intensity are inversely correlated, but it

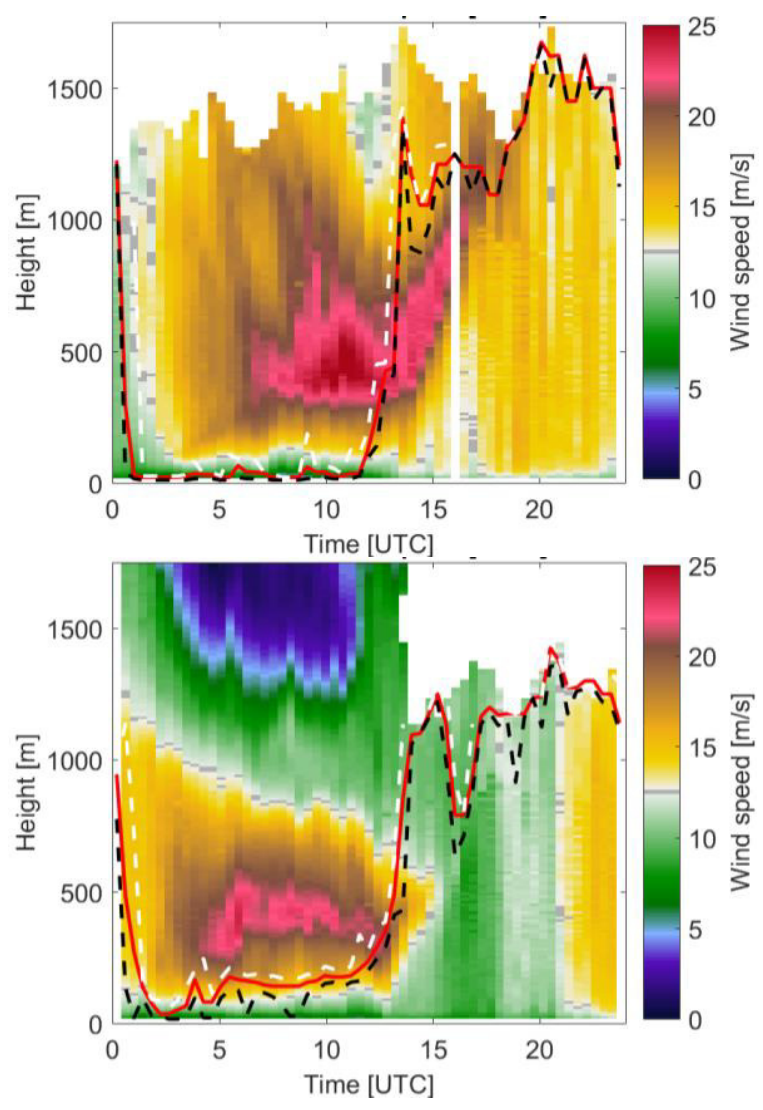

Figure 3: Wind speed from Doppler lidar for the 24 hours of June $20^{\text {th }}$ (top) and $21^{\text {st }}$ (bottom) 2015. Red line is retrieved $M L H$, with dashed line confidence bounds.

is unknown whether turbulence weakens strong stable boundary layer inversions or if preexisting weak inversions allow for turbulence production

[5]. Potential temperature profiles for stability analysis will be retrieved from radiosondes and AERI, and the bulk Richardson number profiles will be produced.

Fig. 3 also reveals very steep increases in the MLH as the jets begin to dissipate around sunrise, which is at 1130 UTC. On days without a LLJ the convective boundary layer often rises to high daytime values gradually over a few hours. This rapid jump during persistent LLJ events suggests that the sudden input of solar energy and surface buoyancy may act in conjunction with the LLJ wind shear turbulence to quickly enhance turbulence intensity throughout the lowest several hundred meters of the boundary layer. This hypothesis will be investigated further. 
The MLH algorithm and its individual turbulencerelated components will also be used to target the afternoon transition in relation to LLJ formation and characteristics.

It should be noted that neither of the cases in Fig. 3 is completely unique within the dataset. This study will include several other LLJ events.

\section{SUMMARY}

Turbulence associated with LLJs has been a topic of interest in recent years due to linkages with air quality and weather prediction. A new algorithm that detects mixed layer depth from a combination of Doppler lidar retrievals is well-suited to address topics related to LLJ turbulence, with the Doppler lidar simultaneously profiling the jet wind speed.

While high wind shear is present below all observed LLJ noses, it is not the only factor in determining whether substantial mixing will occur. Wind shear above the nose may have a controlling influence on mixing in the nocturnal boundary layer, with higher shear impacting turbulence generation throughout the layer. Static stability will also be investigated as this work continues to determine if strong inversions are suppressing mixing. Critical values for turbulence generation will be pursued for each of these factors, including a study of the Richardson number.

\section{ACKNOWLEDGEMENTS}

Financial support for this work comes from the Joint Center for Earth Systems Technology and NOAA-CREST/CCNY foundation CREST grant NA11SEC481004.3. We also acknowledge PECAN funding from the National Science Foundation award number 1503563 and from NASA.

\section{References}

[1] Delgado, R., S. D. Rabenhorst, B. B. Demoz, R. M. Hoff, 2015: Elastic lidar measurements of summer nocturnal low level jet events over Baltimore, Maryland, J. Atmos. Chem. 72, 311333.

[2] Tucker, S. C., C. J. Senff, A. M. Weickmann, W. A. Brewer, R. M. Banta, S. P. Sandberg, D. C. Law, and R. M. Hardesty, 2009: Doppler lidar estimation of mixing height using turbulence, shear, and aerosol profiles, $J$. Atmos. Ocean. Tech. 26, 673-688.

[3] Higgins, R. W., Y. Yao, E. S. Yarosh, J. E. Janowiak, K. C. Mo, 1997: Influence of the great plains low-level jet on summertime precipitation and moisture transport over the central United States. J. Clim. 10, 481-507.

[4] Klein, P. M., X.-M. Hu, M. Xue, 2014: Impacts of Mixing Processes in Nocturnal Atmospheric Boundary Layer on Urban Ozone Concentrations. Bound.-Layer Meteor. 150, 107-130.

[5] Bonin, T. A., W. G. Blumberg, P. M. Klein, P. B. Chilson, 2015: Thermodynamic and Turbulence Characteristics of the Southern Great Plains Nocturnal Boundary Layer Under Differing Turbulent Regimes. Boundary-Layer Meteorol. 157, 401-420. 